\title{
Approximate symmetry laws for percolation in complex systems: Percolation in polydisperse composites
}

\author{
Alexei S. Ioselevich ${ }^{1,2}$ and Alexei A. Kornyshev ${ }^{1}$ \\ ${ }^{1}$ Institute for Materials and Processes in Energy Systems (IWV-3), Research Center Jülich GmbH, D-52425 Jülich, Germany \\ ${ }^{2}$ Landau Institute for Theoretical Physics, Russian Academy of Sciences, Kosygina Street 2, 117940 Moscow, Russia
}

(Received 24 May 2001; published 9 January 2002)

\begin{abstract}
The concept of so-called global symmetry of percolation models is discussed and extended to multicolored models. An integral equation is obtained, which determines the partial percolation probabilities $P_{a}$ for sites of color $a$. This equation is applied to a polydisperse particulate composite: a mixture of conducting (of relative fraction $x_{m}$ ) and nonconducting spheres with distributions of sizes $n_{m}(R)$ and $n_{i}(R)$, respectively. We find the probability $P_{R}$ for a conducting particle of radius $R$ to belong to the percolation cluster as a function of $x_{m}$ and a functional of $n_{m}\left(R^{\prime}\right)$ and $n_{i}\left(R^{\prime}\right)$. The percolation threshold $x$ is shown to decrease with increasing dispersion $\Delta$ of particle sizes. A simple law $x=1 /(3[1+(\Delta / 4)])$ is obtained in the range of moderate dispersions. The theory is applicable also to a mixture of electronic and ionic conductors.
\end{abstract}

DOI: 10.1103/PhysRevE.65.021301

PACS number(s): 45.70.-n, 81.05.Mh, 72.80.Tm

\section{INTRODUCTION}

The present work is motivated by the need for understanding the properties of technologically important polydisperse composite materials (see [1]), produced by sintering of mixtures of roughly spherical granules. Their structure can be reasonably well reconstructed in a computer simulation (see [2] and references therein). The individual granules can be made of different materials; in this paper we will concentrate on two-component mixtures with granules of two sorts: metallic (conductors) and insulators (or ionic conductors). Two-component conducting composite ceramics have a vast field of application in electronics, electrical engineering, catalysis, and electrical power generation $[1,3]$.

Due to the stochastic character of the formation process, the grains in a composite material do not form a regular lattice but rather a topologically disordered network, whose density is considerably lower than that of close packing [2]. The physical characteristics of the system are closely related to its statistical geometry. For example, in order to find the electrical properties of a two-component metal-insulator composite, one has to address the problem of percolation through the subnetwork of metal grains. The solution depends both on the details of the sintering process and on the distributions $n_{m}\left(r_{m}\right)$ and $n_{i}\left(r_{i}\right)$ of the radii $r_{m}$ and $r_{i}$ of the metal and insulating grains, respectively. In the present paper we adopt a standard model for sintering (see $[2,4,5]$ ) and concentrate on the dependence of the percolative properties of the network on the size distribution of grains.

An ideal case of a binary composite material, where granules of the same sort have the same size, so that $n_{m}\left(r_{m}\right)$ $=\delta\left(r_{m}-R_{m}\right)$ and $n_{i}\left(r_{i}\right)=\delta\left(r_{i}-R_{i}\right)$, has been extensively studied both experimentally [6] and numerically [7-11]; the dependence of the physical properties on the asymmetry parameter $\rho=R_{i} / R_{m}$ is well documented. Still more data are available for the simplest case of equal-size distribution ( $\rho$ =1) $[12-16]$.

In practice, however, there is always a considerable dispersion of the grain sizes. Not much is known about the influence of this dispersion on the properties of the system (see, however, $[8,10])$. Should one try to suppress the dispersion in order to improve the performance of the composite material, or would the size dispersion facilitate it? How can one quantify the effects of dispersion?

The present paper mostly addresses these questions. We propose an approximate analytical approach to the problem, based on certain approximate symmetries. We demonstrate that moderate dispersion improves the conditions for percolation, and find explicit dependences of the percolation threshold and percolation probability on the dispersion. We also give a qualitative explanation of these dependences.

Polydisperse composite materials are complex systems; a reliable quantitative characterization of their percolative properties is feasible only with the aid of massive numerical simulation. All the characteristics of the system are functionals of the grain-size distribution functions $n_{m}\left(r_{m}\right)$ and $n_{i}\left(r_{i}\right)$. Therefore, in order to conduct a comprehensive numerical study of the problem, one has first to choose a discrete set of trial functions for $n_{m}\left(r_{m}\right)$ and $n_{i}\left(r_{i}\right)$ and then determine (by means of numerical simulation) the characteristics of the statistical geometry for each choice. This task would require both huge numerical resources and a certain idea a priori about which set of trial functions would be most efficient. If we want to optimize the structure of the composite for a certain goal, we will then need to extend the results to the continuous space of distributions. All these complications make the problem difficult for $a b$ initio numerical study and suggest the need for some approximate analytical framework. Certainly, $a b$ initio analytical treatment of the problem is impossible (it is impossible even for much simpler systems). Instead, we propose a semiphenomenological procedure of finding the properties of a certain class of systems (characterized by the distribution functions of the radii) provided these properties are known for one simple representative of the class - a system of spheres with equal radii. This task turns out to be feasible due to a certain (approximate) symmetry observed in the numerical simulations [9].

Although approximate, this symmetry appears to be general for many percolation models. In its truncated form 
(which we will call "local") such symmetry has been known from the early work on percolation theory in the 1960s, and it has the following meaning.

There are various classes of percolation models. Each model of a given class is characterized by a set of parameters $\{\mu\}$ and undergoes a percolation transition at certain values of these parameters. Within each class there should exist a certain function of the parameters-the invariant $I\{\mu\}$-which takes, at the percolation transition, the same value $I_{c r}$ for all the models of the class. This statement by itself is trivial, as it simply states the existence of the surface of percolation transitions in the space of parameters $\{\mu\}$. The nontrivial observation is that $I\{\mu\}$ strongly depends on only a few relevant parameters and is almost independent of all other-irrelevant-parameters. For example, for lattice bond-percolation models the invariant $I$ depends on the probability of bond formation $p$, on the coordination number $Z$, and on the dimensionality of the lattice $d$, but it is approximately independent of the type of lattice. The "symmetry" is nothing other than the above approximate independence of $I$ on certain characteristics of the model. Its accuracy is usually of the order of a few percent.

A detailed analysis shows, however, that the above "local symmetry" can actually be extended to a "global symmetry." That is the numerical data show that not only is the value of $I\{\mu\}$ at the percolation threshold approximately invariant, but also the percolation probability $P$ takes approximately the same values for different models of the class, provided that these models are characterized by the same values of $I$. In other words, each class is characterized by an invariant function $P(I)$. The global symmetry claims that the percolation probability $P$ is a function of a single variablethe invariant $I$-and this is true within the entire space of parameters $\{\mu\}$, not only in the vicinity of the percolation threshold. This is fulfilled with an accuracy similar to the accuracy of the local symmetry, so that there is no reason to use the local symmetry without taking advantage of the broader global one.

The generalization of the global symmetry to colored models, where each species $a$ is characterized by its own percolation probability $P_{a}$, is not obvious. It can, however, be accomplished in a similar way as for the local symmetry case in [17], where an approximate criterion was proposed for percolation in a system with an arbitrary number of different species- "colors." In the present work we generalize the arguments of [17] to validate the global symmetry. This generalization allows one to find not only the percolation threshold, but also the percolation probability and distribution of sites within the percolating cluster over different species, which is important for applications. These characteristics can be compared with the results of experiments and numerical simulations.

We should stress that the approximate generalized theory of global symmetry is not devised to describe the delicate universal behavior of the system near the percolation threshold (critical exponents, etc.). It rather claims the ability to treat a reasonably wide range of complex systems, characterized by a multitude of parameters, with the help of relatively simple universal formulas.
The paper is organized as follows. In Sec. II we review the local symmetries known for different percolating systems, with the emphasis on the colored topologically disordered models, to which class the polydisperse composite materials belong. In Sec. III we introduce the concept of global symmetry and demonstrate its existence for cases when the local symmetry exists. Again, the colored models are discussed most extensively. The equation of state governing the partial percolation probabilities for different species is introduced and the properties of its solutions are described. In Sec. IV we apply the general methods to particulate composites and find the dependence of the percolation threshold and partial densities of the percolation cluster on the parameters of the particle size distributions. A summary of the main results and a discussion of the limitations of the present theory conclude the paper.

\section{LOCAL SYMMETRIES IN PERCOLATION MODELS: AN OVERVIEW}

The idea of what we call "local symmetry" is by no means new, so we start our discussion with a review of different types of local symmetry, found in different systems.

\section{A. Percolation on a regular lattice}

The local symmetry was first proposed by Domb and Sykes $[18]$ (see also $[19,20]$ ), who noticed that for all bondpercolation problems on various regular lattices of a given dimensionality $d$ the percolation threshold $p_{c r}$ is, roughly, inversely proportional to the coordination number $Z$. In other words, if one considers the average number $B=Z p$ of bonds connecting a given site to its neighbors, then the value of $B$ at the percolation threshold, $B_{c r}$, is approximately invariant over the entire class of bond-percolation problems of fixed dimensionality: $B_{c r}(d) \approx d /(d-1)$.

Later, Scher and Zallen [21] proposed a different invariant-the "volume fraction" $\vartheta$-for the site percolation. It is constructed as follows. One draws identical spheres around all sites of the lattice in such a way that the spheres corresponding to neighboring sites touch each other. Then one calculates the density of packing $\rho_{p}$ : the ratio of the total volume of all spheres to the entire volume of the lattice. The invariant is $\vartheta=\rho_{p} x$, where $x$ is the probability for a given site to be "painted black" (if one considers percolation via black sites). Thus, $\vartheta$ has the meaning of the packing density for the subsystem of "black spheres" in the above construction. Again, the value $\vartheta_{c r}$ of $\vartheta$ at the percolation threshold is approximately invariant for different lattices of the same dimensionality.

The approximate invariance of $\vartheta_{c r}$ and $B_{c r}$ is illustrated in Figs. 1(a),(b). More details about percolation in regular lattices can be found in the classical review by Shante and Kirkpatrick [22].

\section{B. Percolation on random sites}

Skal and Shklovskii [23] and Pike and Seager [24] introduced the invariant for the model of percolation on random sites (also called the "Swiss cheese model;" see [25]). In this 

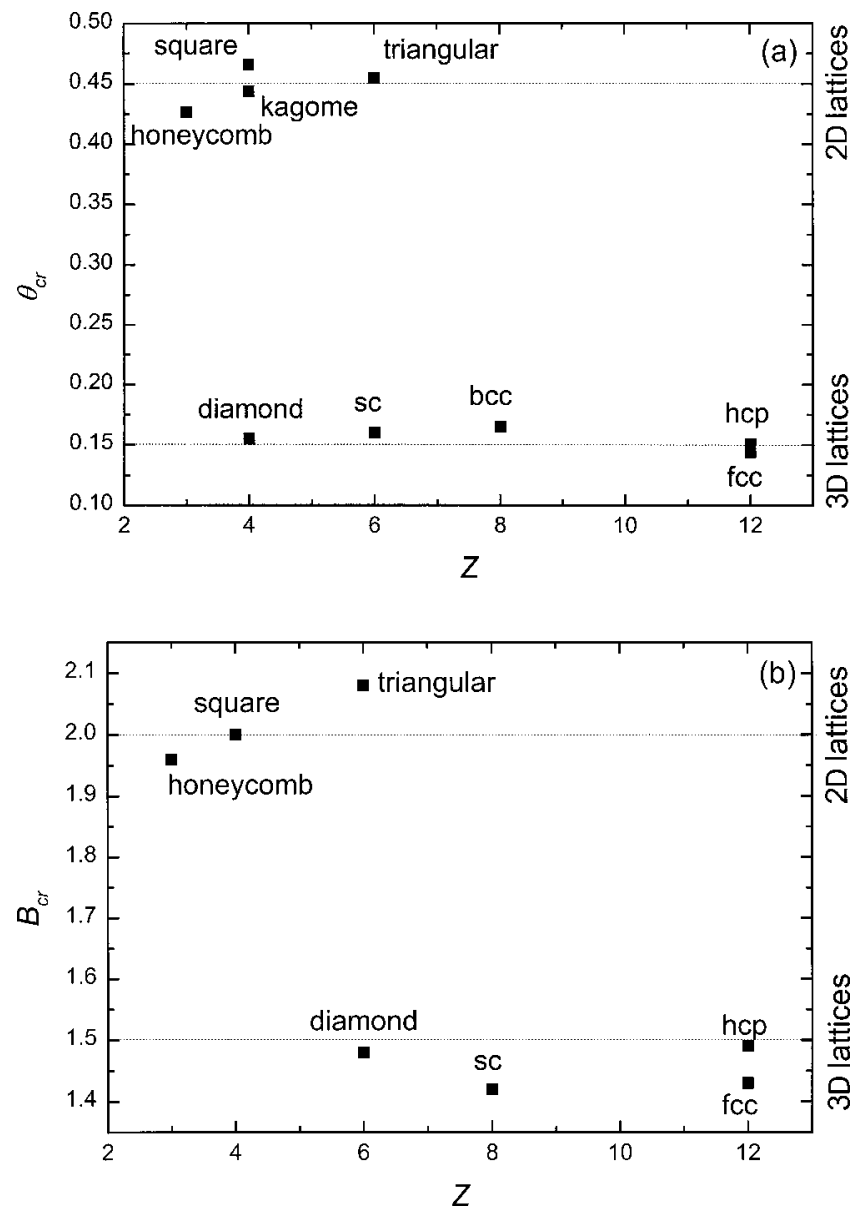

FIG. 1. Illustration of local symmetry for percolation models on different two- (2D) and three-dimensional (3D) lattices (after [22]). $Z$ is the coordination number. (a) Values of $\vartheta_{c r}$ for site percolation; (b) values of $B_{c r} \equiv Z p_{c r}$ for bond percolation.

problem one considers a medium with randomly placed identical cavities (random sites), usually-but not necessarily — of spherical shape. Percolation occurs when an infinite number of holes overlap, so that one can travel throughout the system via an infinite labyrinth of connected cavities. This model is essential for the theory of hopping conductivity in semiconductors; see [26]. It is interesting that the physical meaning of the invariant $B$ for the class of random-site-percolation models appears to be exactly the same as for the bond-percolation class: it is the average number of holes overlapping with any given one. The local symmetry is that the value of $B$ at the percolation threshold is approximately independent of the shape of the individual cavity. However, the value of $B_{c r}(d)$ for the class of topologically disordered random-site-percolation models $\left[B_{c r}(d\right.$ $=2) \approx 4$ and $\left.B_{c r}(d=3) \approx 2.7\right]$ differs considerably from that for bond percolation on regular lattices.

\section{Randomly packed spheres: Binary mixtures}

Consider a mixture of spheres of two sorts: $m$ (metal) with radii $R_{m}$, and $i$ (insulator) with radii $R_{i}$. Since all the metallic spheres in such a mixture are identical, one can apply the ideas of local symmetry to this system also. It was sug- gested $[3,27,28,14,16]$ that this system belongs to the class of $B$-invariant systems, so that the average number $B_{m m}$ of bonds connecting a given metal site to other metal sites is the invariant. Decisive support for this view came from the work by Bouvard and Lange [9]. They simulated the binary composite system and showed numerically that the onset of percolation in the system occurs at the same value of the metalmetal coordination number $B_{m m}=B_{c r} \approx 2$ for different values of the parameter $\rho=R_{m} / R_{i}$ in the range $1 / 3<\rho<3$. Outside this range the accuracy of the local symmetry becomes poor. Hence, it is likely that all binary mixtures with not very strongly differing sizes of constituent grains obey the local symmetry with respect to the invariant $B_{m m}$.

\section{Bond-percolation type of symmetry for topologically disordered site-percolation models}

Both random-packing percolation and random-site percolation demonstrate symmetry described by an invariant of $B$ type. Why do these manifestly site-percolation systems possess symmetry of bond-percolation type? Here one may put forward the following qualitative arguments (see [29]). Let us concentrate on the conducting granules and keep track of the bonds between them. Due to the random environment inherent in the topologically disordered lattice, these bonds, as in the bond-percolation model, are being established roughly independently of each other. The same arguments are also applicable to random-site percolation.

This feature distinguishes the topologically disordered site-percolation model from the conventional site-percolation one (where there is a strong correlation between different bonds of a given site), and makes it rather similar to bond percolation.

\section{E. Colored systems}

The problem of constructing the invariant becomes more complicated if one considers a colored system (see, e.g., [30]), i.e, a system where each site is characterized by a parameter (or a set of parameters) $a$, with a priori probabilities $n_{a}\left(\Sigma_{a} n_{a}=1\right)$. The bonding probability (i.e., the probability $p_{a a}$, for two neighboring sites to be connected) depends on the values of the parameters $a, a^{\prime}$ for the sites involved. As in the conventional site-bond-percolation problem (see [25]), there are two different types of disorder: the sites are randomly occupied by different species, and the bonds between sites are also established randomly. An important complication, however, is the nontrivial character of the matrix $p_{a a^{\prime}}$ : the bonds are not necessarily established only between sites of the same color. In topologically disordered systems the picture is further complicated due to fluctuations of the local coordination.

The class of colored percolation problems is extremely wide: for example, it includes both bond percolation and site percolation as limiting subclasses. Thus, the general invariant $I$ for the colored percolation class (provided it exists) must reduce in the two limits to $B$ and $\vartheta$, respectively. The character of local correlation in these two subclasses is, however, very different. Therefore finding a reasonable interpolation between $B$ and $\vartheta$ is a tough problem: to our knowl- 
edge, nobody has solved it so far. In [17], and also in the present paper, we restricted our consideration to the domain of "bond-percolation-type problems" by postulating that the invariant for the problem of interest is still related to bonding; whether it is true or not for any particular class of systems can only be checked a posteriori. However, having in mind the arguments of the previous subsection, one may expect that the symmetry for topologically disordered colored systems (such as polydisperse two-component mixtures) will again correspond to $B$ type.

The choice of bondlike percolation does not yet fix the structure of the invariant: the way to introduce the bonding invariant $B$ is not unique; to choose the right one we have to invoke additional physical arguments.

\section{The bonding matrix}

Consider an average number $B_{a a^{\prime}}$ of bonds connecting a given site of color $a$ to other sites of color $a^{\prime}$. It can be written in the form

$$
B_{a a^{\prime}}=b_{a a^{\prime}} n_{a^{\prime}} .
$$

In the case of colored percolation on a regular lattice with fixed coordination number $Z$ the bonding matrix $\hat{b}$ does not depend on the distribution function $\vec{n}$; it is simply related to the bonding probability: $b_{a a^{\prime}}=Z p_{a a^{\prime}}$. In the present paper we are mostly concerned with percolation in topologically disordered networks, where $Z$ itself is subject to fluctuations, so it seems more appealing to introduce $b_{a a^{\prime}}$ as a primary characteristic of the system, comprising both fluctuations of bonding and fluctuations of coordination. It is a tricky point, however. Indeed, in random networks the local coordination may depend on the coloring of the environment (especially if the "color" reflects the size of a granule occupying the site), and therefore $\hat{b}$ may itself be a functional of $\vec{n}$. Such an effect of correlation between the bonding matrix and the distribution function does exist in the system of randomly packed spheres with which we are concerned. Fortunately, for not very wide distributions (such that relevant radii in the distribution differ by not more than a factor of 3) it has a trivial form: the distribution function enters $\hat{b}$ only through the average squared grain radius $R^{2}$ (see Sec. IV). For broader distributions the correlations become nontrivial, and our theory should be considerably modified in this case. We have so far not been able to elucidate the character of these correlations and make necessary modifications.

Thus, the concept of the bonding matrix is meaningful and productive for some models (we will call them the bonding matrix models), while for other models the formula (1) does not make much sense, since $\hat{b}$ depends on $\vec{n}$ in some unknown way. The general approach developed below is effective only when applied to bonding matrix models.

\section{Construction of invariant}

The total average number of bonds for a site of color $a$ is

$$
B_{a}=\sum_{a^{\prime}} b_{a a^{\prime}} n_{a^{\prime}}
$$

Since $b_{a a^{\prime}}$ is a matrix, one has yet to propose a procedure for producing the invariant $B$ (which needs to be a scalar) out of this matrix. This task turns out to be a nontrivial one. The simplest "naive" version would be just to average $B_{a}$ over the colors $a$ with certain properly chosen weights. No clear procedure for choosing these weights has been proposed. Some ad hoc prescriptions (see, e.g., [26]) are logically questionable; furthermore, they work poorly. Why do these attempts fail? Apparently the reason is as follows. Since the sites are not equivalent in the colored problem, the bonds should be counted with certain color-dependent weights, which reflect the effective ability of a given neighbor to be further connected to the infinite cluster. And that should already be done when calculating $B_{a}$ for a fixed color $a$, not only at the stage of averaging it over colors. A self-consistent prescription for assigning the weights for different colors was proposed in [17]. The key idea is that the weights should reflect the participation of a given color in the infinite percolation cluster. Certain colors are more likely to be bonded, therefore their relative representation in the infinite cluster is enhanced, compared to their a priori probability, while some other colors are bonded less readily, and they should be less represented in the infinite cluster. Thus, the probabilities $n_{a}^{(i . c .)}$ of finding given species within the infinite cluster depend on the structure of the bonding matrix; they should be found self-consistently.

The procedure proposed in [17] for finding $n_{a}^{(i . c .)}$ is based on the assumption, that, at least in the vicinity of the percolation threshold, the partial percolation probability $P_{a}$ $\equiv n_{a}^{(i . c .)} / n_{a}$, i.e., the probability for a given site of color $a$ to belong to the infinite cluster of bound sites, depends on one single parameter, characterizing its average local coordination, namely, on the average number $B_{a}^{(i . c .)}$ of relevant bonds, defined as those bonds that connect it with an infinite cluster:

$$
B_{a}^{(i . c .)}=\sum_{a^{\prime}} b_{a a^{\prime}} n_{a^{\prime}}^{(i . c .)}=\sum_{a^{\prime}} b_{a a^{\prime}} n_{a^{\prime}} P_{a^{\prime}} .
$$

Thus, in [17] it was postulated that

$$
P_{a}=W\left(B_{a}^{(i . c .)}\right),
$$

where the function $W(B)$ is universal and independent of $a$ for a given class of systems, at least in the vicinity of the percolation threshold. Note, that, in contrast to the a priori colorings of sites, the partial densities of the infinite percolation cluster are strongly correlated, especially near the percolation transition. For example, the probability $n_{a_{1} a_{2}}^{(i . c .)}\left(i_{1} i_{2}\right)$ of finding both sites $i_{1}$ and $i_{2}$ belonging to the infinite cluster and additionally having colors $a_{1}$ and $a_{2}$ does not reduce to a product of $n_{a_{1}}^{(i . c .)}$ and $n_{a_{2}}^{(i . c .)}$. However, by definition, the nontrivial correlators do not enter the average number of relevant bonds $B_{a}^{(i . c .)}$ and the formula (3) is exact. Thus, the crucial approximation is the assumption that $P_{a}$ depends exclusively on $n^{(i . c .)}$, not on higher correlators, and, moreover, that it depends on $n^{(i . c .)}$ only through $B_{a}^{(i . c .)}$. The solution of the system of Eqs. (3),(4) gives, in principle, the partial per- 
colation probabilities $P_{a}$ and the percolation threshold as the point where all $P_{a}$ simultaneously vanish.

\section{The percolation threshold}

Near the percolation threshold the percolation probabilities are small; therefore the argument $B_{a}^{(i . c .)}$ of the function $W\left(B_{a}^{(i . c .)}\right)$ should also be small. In the spirit of the general mean field theory of phase transitions we postulate an analytic behavior of the function $W(B)$ at small $B$ :

$$
W(B)=\frac{B}{B_{c r}}-\text { const } \times B^{2}+\cdots .
$$

The coefficient in front of the quadratic term should be negative to ensure that the percolation exists for $B>B_{c r}$ and is absent for $B<B_{c r}$. The percolation criterion can easily be found as the condition that Eqs. (3),(4) have a nontrival solution. As a result, this criterion reads

$$
B\{\vec{n}, \hat{b}\}=B_{c r}
$$

where the self-consistently weighted invariant $B\{\vec{n}, \hat{b}\}$ is the maximal eigenvalue of a symmetric real matrix

$$
\tilde{b}_{a a^{\prime}}=\sqrt{n_{a} n_{a^{\prime}}} b_{a a^{\prime}} .
$$

Equation (6) defines a surface of percolation transitions in the space of parameters $\vec{n}$ (vector in color indices) and $\hat{b}$ (matrix in color indices). Each point in this space represents a particular system of the class, where the local symmetry is presumed.

The generalized percolation criterion (6), first introduced in [17], appears to be quite successful in some cases. For example, it made it possible to describe the temperature dependence of the hopping conductivity in the whole range of temperatures by a universal formula with a single parameter (the critical value $B_{c r}$ ): both low and high temperature limiting regimes were reproduced with high accuracy by a single formula (see [17]). One should not forget, however, the approximate character of the symmetry. Any theory based on it might be inaccurate or even misleading, if systems with very different properties are forcefully included in the same class.

\section{Partial percolation probabilities near the percolation threshold and critical modes}

To study the color dependence of $P_{a}$ near the threshold it is convenient to expand it in terms of normalized eigenvectors $\psi_{a}^{(\alpha)}$ of the matrix $\widetilde{b}_{a a^{\prime}}$ :

$$
P_{a}=\frac{1}{\sqrt{n_{a}}} \sum_{\alpha} u_{\alpha} \psi_{a}^{(\alpha)},
$$

where $u_{\alpha}$ are some amplitude coefficients yet to be found. The eigenvector related to the maximal eigenvalue we will call the critical mode and denote it as $\Psi_{a}$ for brevity; the corresponding critical amplitude is $U$. Analysis of Eqs. (3),(4) taking account of the quadratic correction to $W$ shows that all the amplitudes $u_{\alpha}$ simultaneously vanish, as $B$ approaches $B_{c r}$ from above. However, the critical amplitude vanishes as a linear function of $B-B_{c r}$, wnile noncritical amplitudes vanish much faster. Thus, in full accord with the general theory of second order phase transitions, only the critical mode survives in the vicinity of the transition. Finally, we can write the following formula, valid in the vicinity of the percolation transition surface:

$$
\begin{aligned}
P_{a}\{\vec{n}, \hat{b}\} \approx & C\{\vec{n}, \hat{b}\} \frac{\Psi_{a}\{\vec{n}, \hat{b}\}}{\sqrt{n_{a}}}\left(B\{\vec{n}, \hat{b}\}-B_{c r}\right)^{\beta} \\
& \times \theta\left(B\{\vec{n}, \hat{b}\}-B_{c r}\right),
\end{aligned}
$$

where $\beta$ is the exponent for the infinite cluster density. If the above analysis [based on the assumption of an analytic behavior of $W(B)]$ is taken seriously, the critical exponent $\beta$ $=1$, which is the well known mean field result. Fluctuations strongly renormalize the amplitude $U$ in the critical region, changing the critical exponent $\beta$. At the same time, the critical fluctuations do not affect the shape of the critical mode $\Psi_{a}$. Thus the formula (9), with properly renormalized exponent $\beta$, remains valid in the critical regime also. As long as the unique critical mode is singled out in the vicinity of the transition, the colored (i.e., multimode) nature of the initial model becomes irrelevant for its critical behavior. Therefore the true (renormalized) critical exponents (in particular, $\beta$ ) for the colored percolation models should have standard values-the same as for classical single-color percolation models (see [25]). The scalar functional $C\{\vec{n}, \hat{b}\}$, though unknown, is nonsingular at the percolation surface. It plays the role of the nonuniversal constant of the standard theory of critical phenomena.

\section{THE GLOBAL SYMMETRY}

The symmetry discussed in the previous section concerned only the position of the surface of percolation transitions, and the percolation probability in its close vicinity. Now we try to go further and examine the percolation probabilities in the whole range of parameters.

Let us take for orientation the percolation probabilities $P$ obtained by Frisch et al. [31]. In Fig. 2(a) we plot $P$ for the site-percolation problem, not as a function of the site concentration $x$, but as a function of the invariant $\vartheta$. One can see that the curves for different lattices of the same dimensionality $d$ but differing in $Z$ merge with the same accuracy of a few percent, not only at the threshold, but in the whole range of $\vartheta$ 's. This observation means that, without lowering the accuracy of approximation, we can not only postulate the same values of $\vartheta_{c r}$ for all these lattices, but assume that the entire function $P(\vartheta)$ is identical for them. This assumption we will refer to as global symmetry, in contrast to local symmetry, the latter claiming only coincidence of percolation thresholds $\vartheta_{c r}$.

For the case of the bond-percolation model the assumption of global symmetry does not work as well as for site percolation. In Fig. 2(b) we plot $P$ for bond percolation (also 

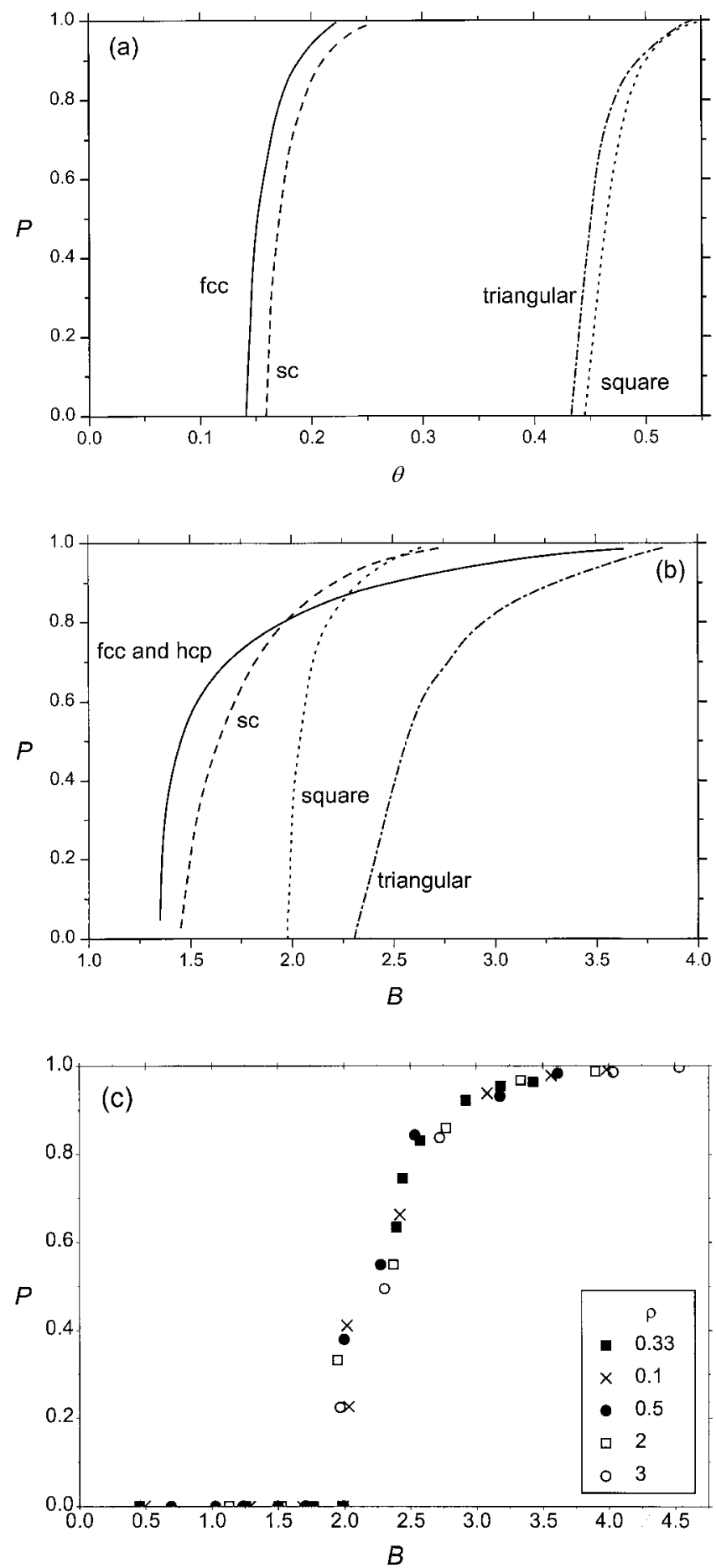

FIG. 2. Illustration of global symmetry for percolation in different systems. (a) Percolation probability $P$ for site percolation on different 2D and 3D lattices (after [31]), replotted against $\vartheta$. (b) The same for bond percolation, replotted against $B$. (c) The same for a binary mixture of metal spheres of radius $R_{m}$ and insulating ones of radius $R_{i}$ (after [9]) plotted against $B=B_{m m}$ (the average metal-metal coordination number), for different values of the parameter $\rho \equiv R_{i} / R_{m}$.

taken from [31]) as a function of the invariant $B=Z p$. One can see that the curves for different 3D lattices are not that far from each other, and for crude estimations in 3D one can postulate global symmetry for lattice bond percolation. For 2D lattices the curves do not merge at all: the global symmetry does not hold.

Global symmetry has been observed in a binary system of randomly packed spheres. Bouvard and Lange [9] numerically found both the percolation probability $P$ for a metal grain and the average metal-metal coordination number $B_{m m}$. Then they plotted $P$ against $B_{m m}$. The plots obtained for different values of the parameter $\rho=R_{m} / R_{e}$ in the range $1 / 3<\rho<3$ merged perfectly, which suggests global symmetry for a class of binary mixtures with reasonably broad size distributions [see Fig. 2(c)].

In general, the global symmetry for classes of single-color percolation models can be formulated as follows. Each class of (approximate) symmetry is characterized by the form of the invariant $I\{\mu\}$ and a function $\mathcal{P}(I)$. The percolation probability for any system with parameters $\{\mu\}$ is then $P$ $\approx \mathcal{P}(I\{\mu\})$. The above analysis has demonstrated that the global symmetry works perfectly well for percolation in randomly packed binary mixtures and for site percolation on regular lattices. For the bond percolation on 3D lattices the global symmetry works reasonably well, while for bond percolation on 2D lattices it seems to break down. Note, however, that for 2D bond percolation already the local symmetry is much worse than in other cases.

\section{A. Global symmetry for colored systems}

Since, in the case of colored systems, there is no unique percolation probability, but only partial percolation probabilities $P_{a}$ for different colors, the generalization of the concept of global symmetry for colored systems is not straightforward.

\section{The equation of state}

The most natural generalization rests on the assumption that Eq. (4) is valid not only in the vicinity of the percolation threshold but everywhere. Thus, we postulate the "equation of state"

$$
P_{a}=W\left[\sum_{a^{\prime}} b_{a a^{\prime}} n_{a^{\prime}} P_{a^{\prime}}\right] .
$$

This nonlinear integral equation governs the partial percolation probabilities $P_{a}$.

\section{Universality of the structure function $W(x)$}

The structure function $W(x)$, appearing in the equation of state (10), plays a crucial role in our approach. It is supposed to be approximately system independent, within a certain reasonably broad class of systems; i.e., it depends neither on the color distribution parameters $\vec{n}$ nor on elements of the bonding matrix $\hat{b}$. It reflects, however, the topology of the infinite percolation cluster. The universality of the function $W(x)$ means that the topology of the infinite cluster does not change with a change of its color composition. If one adopts the universality of $W$, then the equation of state (10) gives an equally universal solution which determines the dependence 
of the partial percolation probabilities $P_{a}$ on the parameters of the system. Thus, the assumption of universality of the function $W$ is the generalization of the global symmetry assumption for colored systems.

The important consequence of global symmetry in practice is the possibility of extracting the structure function governing the entire class of colored systems from the percolation probability $P(B)$ found (numerically) for a subclass of single-color systems. By definition, in a single-color system there is only one "active" species $a_{0}$, so that bonds can only be formed between sites of color $a_{0}$ (the existence and number of other, "passive" species is irrelevant for the active species bonding pattern). For such a system the matrix $b_{a a^{\prime}}$ reduces to a scalar $b_{a_{0} a_{0}} \equiv b$ and $n_{a}$ has only one relevant component, $n_{a_{0}} \equiv n$. Equation (10) for this case becomes

$$
P=W(b P n),
$$

while the invariant, obviously, is $B=b n$ in this case. Thus, we end up with the equation

$$
P=W(B P) .
$$

On the other hand, the global symmetry for the subclass of single-color systems requires that $P=\mathcal{P}(B)$. As a result, we obtain the following implicit definition for the structure function $W(x)$ in terms of the function $\mathcal{P}(B)$ :

$$
W=\mathcal{P}\left(\frac{x}{W}\right) .
$$

The general behavior of the function $W(x)$ can be derived from that of $\mathcal{P}(B)$; namely, it is easy to show that wellknown properties of the percolation probability,

$$
\begin{aligned}
& \mathcal{P}(B) \equiv 0 \quad \text { if } B<B_{c r}, \\
& \mathcal{P}(B) \rightarrow 1 \quad \text { if } B \gg B_{c r},
\end{aligned}
$$

lead to the following asymptotics of the function $W(x)$ :

$$
W(x) \approx \begin{cases}x / B_{c r} & \text { if } x \ll B_{c r} \\ 1 & \text { if } x \gg B_{c r} .\end{cases}
$$

Indeed, Eq. (13), together with the property $\left.\mathcal{P}(B)\right|_{B \rightarrow B_{c r}}$ $\rightarrow 0$, require that $\left.(x / W)\right|_{W \rightarrow 0} \rightarrow B_{c r}$. This is equivalent to the first line in Eq. (15).

\section{The case of multiplicative bonding matrix}

Let us discuss one special case, important for polydisperse granular systems. Suppose that each color $a$ is characterized by the "bonding activity" $A_{a}$, and that the bonding matrix may be represented in the multiplicative form

$$
b_{a a^{\prime}}=A_{a} A_{a^{\prime}} .
$$

Then from Eq. (3) we get

$$
B_{a}^{(i . c .)}=\lambda A_{a} \quad \text { where } \quad \lambda=\sum_{a} A_{a} n_{a} P_{a}
$$

so that

$$
P_{a}=W\left(\lambda A_{a}\right),
$$

and the integral equation of state is reduced to a nonlinear equation for one parameter $\lambda$ :

$$
\lambda=\sum_{a} n_{a} A_{a} W\left(\lambda A_{a}\right) .
$$

The self-consistent invariant $B$ entering the percolation criterion,

$$
B=\sum_{a} n_{a} A_{a}^{2}
$$

has the simple meaning of mean squared activity.

\section{APPLICATION OF GLOBAL SYMMETRY APPROACH TO POLYDISPERSE PARTICULATE COMPOSITE MATERIALS}

Powder technology produces metal-insulator and metalsolid-electrolyte composites, used in supercapacitors and solid-oxide fuel cells. In first approximation these composites may be considered as mixtures of randomly packed spheres.

Prompted by results of Bouvard and Lange [9], we adopt the hypothesis of global symmetry for such mixtures. Moreover, we are going to extend it to polydisperse systems, using the general results of the previous section. Such an extension is, certainly, a hypothesis, which has yet to be proved by numerical simulations.

For definiteness we consider a dual system composed of electronically conducting (metal or semiconductor) and electronically nonconducting (insulator or ionic conductor) components. For brevity, they will be called, hereafter, "metal" and "insulator," respectively. In the problem of percolation through the metal subnetwork only the metal grains are active sites, so that it is convenient to concentrate on the metal subnetwork. Although the insulating grains are not sites of this subnetwork, they are certainly important for the probability of establishing bonds between metal sites. Thus, the characteristics of the insulating component will influence the bonding matrix for the metallic subnetwork. The metal grains in the polydisperse mixture are not equivalent: they have different radii. In terms of the general theory of the preceding sections, the radius of a grain plays a role of color. Our first task is then to find out if this system can be described by the bonding matrix model, and, if yes, to elucidate the structure of the matrix.

\section{A. A model for a particulate composite}

We take a standard steepest descent model of the composite deposition process $[2,4,13,15,16,6,9]$. In this model granules fall down into a vessel one by one from random places above (see Fig. 3). They hit the pile of grains, those that have fallen before, most probably at a single point. Although the collision is assumed perfectly inelastic, a configuration in 


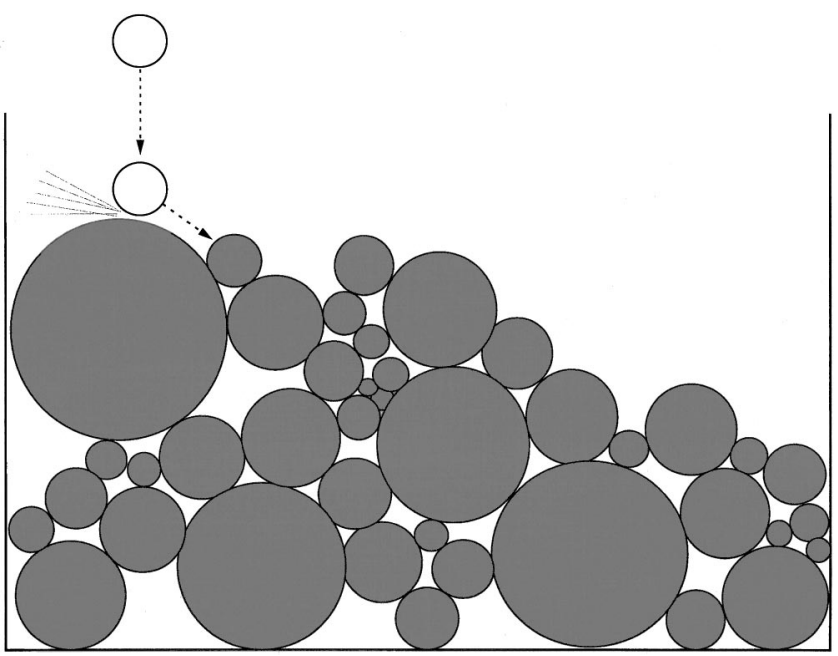

FIG. 3. Illustration for the steepest descent sedimentation process (see text).

which a newcomer grain touches the others at only one point is unstable mechanically. The newcomer thus slides downward along the steepest descent path on the pile surface, until it touches the grains of the pile at three points. Here the sliding stops. These three contacts fix the position of the granule, and it is assumed to remain in this position, not affected by any new granules that could possibly fall on top of it later.

In this idealized model the packing geometry is not affected by the type of grain (metal or insulator). We thus may treat the grains of both types on an equal footing and introduce an overall grain-radius distribution (irrespective of the grain sort)

$$
n(r)=x_{m} n_{m}(r)+x_{i} n_{i}(r),
$$

where $x_{m}$ and $x_{i}$ are the particle fractions of metal and insulator, respectively,

$$
x_{m}+x_{i}=1
$$

It is also convenient to introduce three different types of averaging: the averagings over size distributions of metal or insulating grains separately,

$$
\begin{aligned}
& \langle(\cdots)\rangle_{m} \equiv \int_{0}^{\infty}(\cdots) n_{m}(r) d r, \\
& \langle(\cdots)\rangle_{i} \equiv \int_{0}^{\infty}(\cdots) n_{i}(r) d r,
\end{aligned}
$$

and overall averaging,

$$
\langle(\cdots)\rangle \equiv \int_{0}^{\infty}(\cdots) n(r) d r=x_{m}\langle(\cdots)\rangle_{m}+x_{i}\langle(\cdots)\rangle_{i} .
$$

\section{B. Statistics of coordination for a particulate composite}

Every new grain adds exactly three new intergrain contacts to the system. Since each contact connects two granules, we conclude that the average number of contacts of a granule is

$$
Z_{0}=3 \times 2=6
$$

The next step is to determine $Z(r)$ - the number of contacts with arbitrary other grains that, on average, a grain of radius $r$ has. A priori we know only that $Z(r) \geqslant 3$, because each grain has at least three contacts, required by mechanical stability.

A plausible assumption, proposed in Ref. [9] (see also the earlier work $[7,5])$, is that the excess $Z(r)-3$ is proportional to the surface of the particle; thus

$$
Z(r)-3=A r^{2}
$$

where the constant $A$ can be found from the normalization condition

$$
\langle Z\rangle=Z_{0}=6
$$

As a result, one obtains

$$
Z(r)=3\left(1+\frac{r^{2}}{R^{2}}\right),
$$

where

$$
R^{2}=\left\langle r^{2}\right\rangle=x_{m} R_{m}^{2}+x_{i} R_{i}^{2}
$$

is the overall average squared radius of the grains $\left(R_{m}^{2}\right.$ $\equiv\left\langle r^{2}\right\rangle_{m}$ and $R_{i}^{2} \equiv\left\langle r^{2}\right\rangle_{i}$ being the average squared radii of metal and insulating grains, respectively). Although the ansatz (26) has never been justified theoretically, numerical simulations solidly support it, at least in the case when the distribution of sizes is not too broad.

It is, however, not enough to know $Z(r)$ for determination of the system properties. One needs to know how the partners contacting a given grain of radius $r_{1}$ are distributed over radii $r_{2}$. Such a distribution $Z\left(r_{1}, r_{2}\right)$ should obey the obvious normalization condition

$$
\int_{0}^{\infty} Z\left(r_{1}, r_{2}\right) d r_{2}=Z\left(r_{1}\right) .
$$

Yet another ansatz, a mutiplicative one, also put forward in [9] (see also [32]), fixes the functional form of $Z\left(r_{1}, r_{2}\right)$. In a slightly modified form-to fit our polydisperse case-it is

$$
\frac{Z\left(r_{1}, r_{2}\right)}{Z\left(r_{1}\right)} \propto n\left(r_{2}\right) Z\left(r_{2}\right),
$$

which means that the fraction of contacts that link any fixed grain with other grains of given size $r_{2}$ is proportional to both the concentration $n\left(r_{2}\right)$ and the activity $Z\left(r_{2}\right)$ of the latter. 
Substitution of (31) in the normalization condition (30) gives the proportionality constant. As a result, one obtains

$$
\begin{aligned}
Z\left(r_{1}, r_{2}\right) & =Z\left(r_{1}\right) Z\left(r_{2}\right) n\left(r_{2}\right) / Z_{0} \\
& =\frac{3}{2} n\left(r_{2}\right)\left(1+\frac{r_{1}^{2}}{R^{2}}\right)\left(1+\frac{r_{2}^{2}}{R^{2}}\right) .
\end{aligned}
$$

For interpretation of the multiplicative ansatz (31) it is instructive to have in mind the following picture. Let us represent each grain of radius $r$ by an object with $Z(r)$ "terminals." These terminals tend to couple with similar terminals of other grains. A contact of grains is just a result of such coupling. One can try to view the collection of all terminals of all grains as a sort of homogeneous uncorrelated gas, where each terminal chooses a partner for coupling at random. This picture (certainly it can only be approximately correct) immediately leads to the statistics of contacts described by formula (31).

\section{Percolative properties of a subsystem of metal grains}

Let us now return to the problem of percolation through the metal subnetwork. We have to address the question: What is the number $B\left(r_{1}\right)$ of bonds (i.e., contacts with other metal grains) for a given metal particle of radius $r_{1}$ ? What is the distribution $B\left(r_{1}, r_{2}\right)$ over the radii $r_{2}$ of the partners in the above bonds? Using the formula (32), and substituting in it the concentration of metal paricles $x_{m} n_{m}\left(r_{2}\right)$ instead of the overall concentration $n\left(r_{2}\right)$, we obtain

$$
\begin{gathered}
B\left(r_{1}, r_{2}\right)=\frac{3 x_{m}}{2} n_{m}\left(r_{2}\right)\left(1+\frac{r_{1}^{2}}{R^{2}}\right)\left(1+\frac{r_{2}^{2}}{R^{2}}\right), \\
B\left(r_{1}\right)=\int B\left(r_{1}, r_{2}\right) d r_{2}=\frac{3 x_{m}}{2}\left(1+\frac{r_{1}^{2}}{R^{2}}\right)\left(1+\frac{R_{m}^{2}}{R^{2}}\right) .
\end{gathered}
$$

Thus, we conclude that the problem of percolation in the polydisperse particulate composite material can be reduced to a continuous variant of the bonding matrix model, with

$$
b\left(r_{1}, r_{2}\right)=\frac{3 x_{m}}{2}\left(1+\frac{r_{1}^{2}}{R^{2}}\right)\left(1+\frac{r_{2}^{2}}{R^{2}}\right) .
$$

Moreover, since the bonding matrix (35) is factorized, the results of the corresponding subsection can be directly applied. The activity of a metal grain of radius $r$ is

$$
A(r)=\sqrt{\frac{3 x_{m}}{2}}\left(1+\frac{r^{2}}{R^{2}}\right),
$$

and the partial percolation probabilities are

$$
P(r)=W\left[\tilde{\lambda}\left(1+\frac{r^{2}}{R^{2}}\right)\right] .
$$

The function $W(x)$ is shown in Fig. 4. It was found numeri-

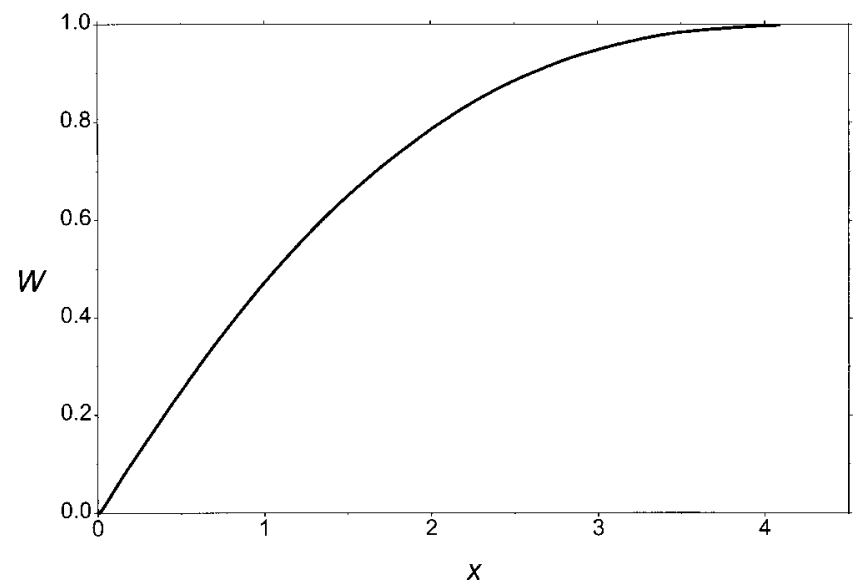

FIG. 4. Plot of the universal function $W(x)$ for the problem of a particulate composite [extracted from the data plotted in Fig. 2(c) with the help of the functional equation (13)].

cally from the functional equation (13) with the $\mathcal{P}(B)$ given by Fig. 2(c). The parameter $\tilde{\lambda}$ in Eq. (37) [its definition here differs from $\lambda$ in Eq. (17) by a factor of $\left.\sqrt{3 x_{m} / 2}\right]$ is an appropriate solution of the nonlinear equation

$$
\tilde{\lambda}=\frac{3}{2} x_{m}\left\langle W\left[\tilde{\lambda}\left(1+\frac{r^{2}}{R^{2}}\right)\right]\left(1+\frac{r^{2}}{R^{2}}\right)\right\rangle_{m}
$$

obtained from Eq. (19). The value of $\widetilde{\lambda}$ depends on the concentration $x_{m}$ and on the distribution functions $n_{m}(r)$ and $n_{i}(r)$ (the latter enters only through $R^{2}$ ).

Application of the percolation criterion (6) with the expression (20) for the invariant $B$ gives

$$
\frac{3}{2} x_{m}\left\langle\left(1+\frac{r^{2}}{R^{2}}\right)^{2}\right\rangle_{m}=B_{c r} .
$$

Expanding the square and rearranging, writing $x_{m}=X$, we arrive at the equation

$$
\begin{aligned}
& \frac{3 X}{2}\left\{\left(1+\frac{1}{X+(1-X) \rho^{2}}\right)^{2}+\frac{\Delta_{m}}{\left[X+(1-X) \rho^{2}\right]^{2}}\right\} \\
& \quad=B_{c r}, \quad B_{c r} \approx 2,
\end{aligned}
$$

which implicitly determines the dependence

$$
x_{m}^{(c r)}=X\left(\rho, \Delta_{m}\right)
$$

of the critical concentration of metal grains $x_{m}^{(c r)}$ on the size distribution functions $n_{m}(r), n_{i}(r)$ through two dimensionless parameters: the asymmetry parameter

$$
\rho^{2}=\left(\frac{R_{i}}{R_{m}}\right)^{2} \equiv \frac{\langle S\rangle_{i}}{\langle S\rangle_{m}}
$$

where $S$ is the area of the grain surface, and the relative dispersion of metal grain surfaces, 


$$
\Delta_{m}=R_{m}^{-4}\left\langle\left(r^{2}-R_{m}^{2}\right)^{2}\right\rangle_{m} \equiv \frac{\left\langle\left(S-\langle S\rangle_{m}\right)^{2}\right\rangle_{m}}{\langle S\rangle_{m}^{2}} .
$$

Note that, to find the critical concentration, one needs to know only the two lowest moments of the surfaces distributions, $\langle S\rangle_{m}$ and $\left\langle S^{2}\right\rangle_{m}$.

In the case of a mixture of two monodisperse components, $\Delta_{m}=0$, and the result (40) reduces to the percolation criterion obtained by Bouvard and Lange [9]. The dispersion of surface areas of metal grains diminishes the value of the threshold $x_{m}^{(c r)}$, i.e., it facilitates percolation. Indeed, for a fixed value of $\rho$ the percolation threshold $x_{m}^{(c r)}$ decreases with increasing $\Delta_{m}$. On the other hand, the dispersion of surfaces of the nonconducting component is irrelevant for $x_{m}^{(c r)}$, as the latter does not depend on $\Delta_{i}$. The effect of dispersion on the percolation threshold is especially transparent in the symmetric case, considered in the next subsection.

\section{The symmetric case}

In many practical cases the distribution functions $n_{m}(r)$ and $n_{i}(r)$ do not differ much, so that an assumption of the same size distribution for both types of particles,

$$
n_{m}(r)=n_{i}(r)=n(r),
$$

may be considered a good approximation. Under this assumption

$$
\rho=1, \quad \Delta_{m}=\Delta_{i} \equiv \Delta,
$$

and Eq. (40) can easily be solved for $X$ :

$$
x_{m}^{(c r)}=X(1, \Delta) \equiv \frac{1}{3(1+\Delta / 4)} .
$$

Thus, in a polydisperse system $(\Delta \neq 0)$ the onset of percolation through the metallic component occurs at a lower concentration of metal than in a monodisperse one $(\Delta=0)$.

What is the physical reason for this effect? To answer this question let us consider a system characterized by the distribution function $n(r)$ and a concentration slightly below the percolation threshold: $x_{m}<x_{m}^{(c r)}\{n(r)\}$. It is easy to show that it is possible to drive the system into the percolating regime by a small variation of the distribution function. Indeed, suppose that we add to the system a small amount of small "doping" particles (with a radius $r_{\text {dop }}$ much smaller that the size $r_{0}$ characteristic for the initial distribution of "matrix" particles); see Fig. 5. The new distribution function $\tilde{n}(r)$ is then given by

$$
\tilde{n}(r)=n(r)+\delta n(r),
$$

where

$$
\delta n(r)=q\left[\delta\left(r-r_{d o p}\right)-n(r)\right],
$$

with small $q$. The doping particles will mostly reside in the pores of the initial network; therefore the direct contacts between the matrix particles will not be affected by doping. On

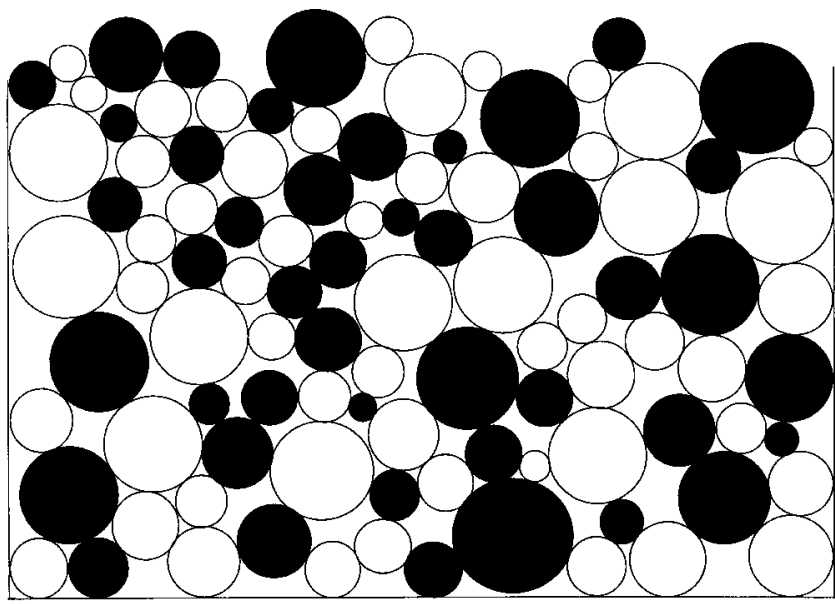

(a)

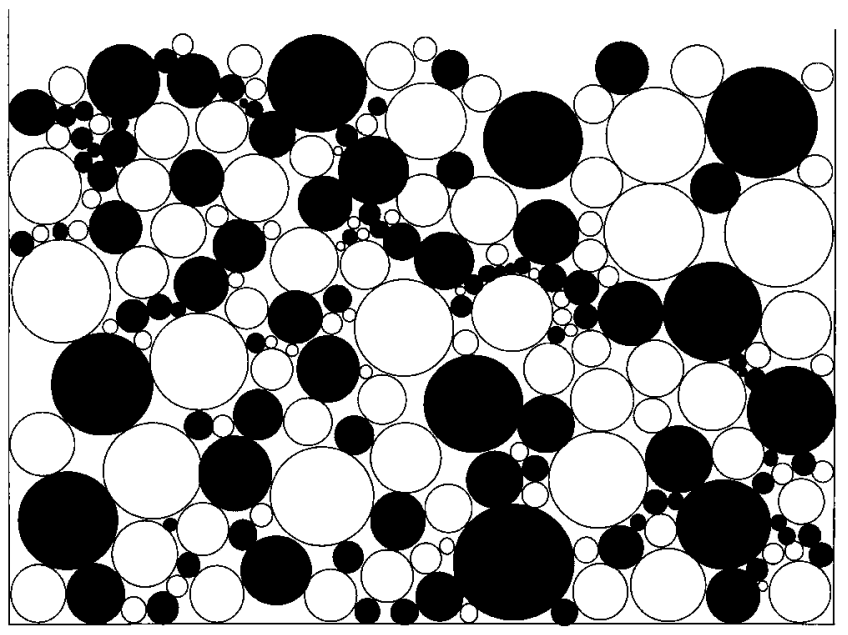

(b)

FIG. 5. Illustration of the question: Why does a diversification of particle sizes facilitate percolation? (a) A pattern with spheres of comparable sizes; (b) the same, after doping with a fraction of much smaller spheres: one can see how additional bonds are established.

the other hand, the small doping particles will establish contacts both with the matrix (most often) and with each other (rarely). As a result, a small but finite number of new indirect contacts between the matrix particles will be established via the doping ones, and this can lead to the onset of percolation. On the other hand, the doping obviously leads to an increase of dispersion:

$$
\Delta \rightarrow \Delta+\delta \Delta, \quad \delta \Delta=q(1+\Delta)>0 .
$$

This case clearly demonstrates a correlation between the lowering of the percolation threshold and increase of the dispersion of particle sizes. The greater the dispersion of particle sizes, the higher the density of the system; in a dense system there is, roughly, a hierarchy of bonds: the bonds between the biggest particles are almost unhindered by the smaller particles, while the latter provide some new bonds; still smaller particles, again, contribute new bonds without affecting the old ones, etc. This idea is illustrated in Fig. 5. 


\section{CONCLUSION}

The most striking practical result of this paper is Eq. (40) for the percolation threshold in polydisperse composites and the rule that follows from it: the greater the dispersion of particle sizes, the lower the percolation threshold. Above the threshold the probability for a particle with size $r$ to belong to the percolation cluster is described by formula (37). For the case of identical size distributions for both components in a mixture, the solution of Eq. (40) gives the simple law Eq. (46). This law, and the basic Eq. (40), are warranted at $\Delta / 4$ $<1$, i.e., they cannot cover orders of magnitude difference of particle sizes. Since the derivation is based on a number of heuristic (although plausible) assumptions, a rigorous validity criterion cannot be obtained from the theory, but should rather be verified by Monte Carlo simulations.

Equations (40) and (37) were derived under certain assumptions which are fulfilled for a model of steepest descent sedimentation (see Sec. IV A). One may envisage other fabrication processes, when these assumptions may not be fulfilled. It would be interesting to investigate whether other fabrication scenarios lead to modification of these equations In all cases, it is most interesting to check the law Eq. (46) for various composites.
[1] Disorder and Granular Media, edited by D. Bideau and A. Hansen (North-Holland, Amsterdam, 1993).

[2] R. Jullien, P. Meakin and A. Pavlovitch, in Disorder and Granular Media (Ref. [1]), Chap. 4, p. 103.

[3] D.S. McLachlan, M. Blaszkiewicz, and R.E. Newnham, J. Am. Ceram. Soc. 73, 2187 (1990).

[4] G. Auvinet, in Proceedings of the Fifth Pan-American Conference on Soil Mechanics and Foundation Engineering, Buenos Aires, 1972 (unpublished).

[5] G. Auvinet and D. Bouvard, in Proceedings of the Fourth International Conference on Application of Statistics and Probability in Soils and Structural Engineering (Pitagora Editrice, Firenze, 1983).

[6] L. Oger, J.P. Troadec, D. Bideau, J.A. Dodds, and M.J. Powell, Powder Technol. 46, 121 (1986); 46, 133 (1986).

[7] R. Ben Aim and P. Le Goff, Powder Technol. 2, 1 (1968).

[8] M. Suzuki and T. Oshima, Powder Technol. 35, 159 (1983).

[9] D. Bouvard and F.F. Lange, Acta Metall. Mater. 39, 3083 (1991).

[10] L.P. Troadec and J.A. Dodds, in Disorder and Granular Media (Ref. [1]), Chap. 5, p. 133.

[11] C.-H. Kuo and P.K. Gupta, Acta Metall. Mater. 43, 397 (1995).

[12] J.P. Fitzpatrick, R.B. Malt, and F. Spaepen, Phys. Lett. 47A, 207 (1974).

[13] A.J. Matheson, J. Phys. C 7, 2569 (1974).

[14] H. Ottavi, J. Clerc, G. Giraud, J. Roussenq, E. Guyon, and
C.D. Mitescu, J. Phys. C 11, 1311 (1980).

[15] M.J. Powell, Powder Technol. 25, 45 (1980); 26, 221 (1980).

[16] M.J. Powell, Phys. Rev. B 20, 4194 (1979); 21, 3725 (1980).

[17] A.S. Ioselevich, Phys. Rev. Lett. 74, 1411 (1995).

[18] C. Domb and M.F. Sykes, Phys. Rev. 122, 77 (1960).

[19] V.A. Vyssotsky, S.B. Gordon, H.L. Frisch, and J.M. Hammersley, Phys. Rev. 123, 1566 (1961).

[20] J.M. Ziman, J. Phys. C 1, 1532 (1968).

[21] H. Scher and R. Zallen, J. Chem. Phys. 53, 3759 (1970).

[22] V.K.S. Shante and S. Kirkpatrick, Adv. Phys. 20, 325 (1971).

[23] A.S. Skal and B.I. Shklovskii, Sov. Phys. Semicond. 7, 1589 (1973).

[24] G.E. Pike and C.H. Seager, Phys. Rev. B 10, 1421 (1974).

[25] D. Stauffer and A. Aharony, Introduction to Percolation Theory (Taylor and Francis, London, 1994).

[26] B.I. Shklovskii and A.L. Efros, Electronic Properties of Doped Semiconductors (Springer, Berlin, 1984).

[27] J. Gurland, Trans. Metall. Soc. AIME 236, 642 (1966).

[28] S.M. Aharoni, J. Appl. Phys. 43, 2463 (1972).

[29] S. Kirkpatrick, Rev. Mod. Phys. 45, 574 (1973).

[30] J.W. Halley, in Percolation Structures and Processes, edited by G. Deutscher, R. Zallen, and J. Adler (Hilger, Bristol, 1983).

[31] H.L. Frisch, J.M. Hammersley, and D.J.A. Welsh, Phys. Rev. 126, 949 (1962).

[32] J.A. Dodds, J. Colloid Interface Sci. 77, 317 (1980). 